\title{
Acute Osteomyelitis in the Hand Due to Dog Bite Injury: A Report of 3 Cases
}

\author{
Ryun Lee, Hee Young Lee, Ji Hyun Kim, Yea Sik Han, Dong Chul Kim, Kwan Chul Tark \\ Department of Plastic and Reconstructive Surgery, Bundang Jesaeng General Hospital, Seongnam, Korea
}

As many people keep small dogs as pets, dog bites are common injuries, accounting for approximately $80 \%-90 \%$ of all animal bite injuries. These injuries usually occur on the upper extremities, most commonly on the fingers. Most of these injuries appear as simple lacerations or abrasions of the skin. Common symptoms include inflammatory reactions of the soft tissue, such as pain, swelling, erythema, and cellulitis. However, the complications of small dog bites may include joint or cartilage injuries, including acute osteomyelitis. Once osteomyelitis develops, it is difficult to diagnose since it has a latency period of approximately 2 weeks. Plain radiography, magnetic resonance imaging, and 3-phase bone scans should be performed when acute osteomyelitis is suspected, and broad-spectrum empiric antibiotic treatment should be administered for approximately 8-12 weeks. We report 3 very rare cases of acute osteomyelitis that occurred after a dog bite injury.

Keywords Osteomyelitis / Magnetic resonance imaging / Bites

\author{
Correspondence: Kwan Chul Tark \\ Department of Plastic and \\ Reconstructive Surgery, Bundang \\ Jesaeng General Hospital, 20 \\ Seohyeon-ro 180beon-gil, Bundang- \\ gu, Seongnam 13590, Korea \\ Tel: +82-31-779-0281 \\ Fax: +82-31-779-5032 \\ E-mail: rulkon@hanmail.net
}

No potential conflict of interest relevant to this article was reported.

Received: 7 Nov 2016• Revised: 28 Apr 2017 • Accepted: 30 May 2017

pISSN: 2234-6163 • elSSN: 2234-6171 • https://doi.org/10.5999/aps.2017.44.5.444 • Arch Plast Surg 2017;44:444-448

\section{INTRODUCTION}

As more people have begun to keep small dogs as pets, dog bite injuries have accordingly become more common, and these injuries account for approximately $80 \%-90 \%$ of all mammalian bite injuries [1-4]. In most cases, these injuries are simple clean lacerations or abrasions on the skin and subcutaneous tissue. As a result, patients and physicians tend to neglect the risk of potential complications [2-4]. These injuries usually occur on the upper extremities, most commonly on the fingers. Common symptoms include inflammatory reactions of the soft tissue, such as pain, swelling, erythema, and cellulitis [4]. The complications of mammalian bite injuries may include joint or cartilage injuries and acute osteomyelitis. However, cases of these complications have rarely been reported in Korea, and especially few cases have been reported of these complications being caused by small dog bite injuries. We report 3 cases of acute osteomyelitis that occurred after a dog bite injury.

\section{CASES}

\section{Case 1}

A 59-year-old female patient visited the emergency center of our hospital with a small $(0.5 \mathrm{~cm}$ long) laceration wound on the distal phalanx (volar and ulnar sides) of her left thumb due to a bite injury by her small pet dog (a Spitz). The patient and dog had both been previously vaccinated against tetanus and rabies respectively. The physical examination showed a simple laceration wound with mild tenderness, and an initial X-ray examination did not show any bony abnormalities (Fig. 1). A simple wound dressing was performed and the patient was discharged with a prescription for an ordinary third-generation oral cephalospo- 
rin. Four days later, the patient revisited our clinic center with noticeable swelling, erythema, and tenderness on her left thumb and stiffness of the interphalangeal thumb joint. She was initially diagnosed with cellulitis on her thumb and admitted after a Gram-stain wound culture was conducted. During her hospitalization, the patient was treated with intravenous systemic antibiotic therapy, including third-generation cephalosporin and aminoglycoside antibiotics. After 1 week of therapy, her inflammatory symptoms had improved and the Gram-stain wound culture did not reveal any bacteria. The patient was discharged with a 1-week prescription of third-generation oral cephalosporin. At her follow-up at our outpatient clinic, the wound condition was found to have worsened, with the development of painful swelling and erythema despite oral antibiotic treatment. Therefore, a follow-up X-ray examination was taken of the left thumb 4 weeks after the injury, and the X-ray images showed focal osteo-

\section{Fig. 1. Initial gross photograph of the wound}

A simple laceration wound with mild tenderness on the distal phalanx of left thumb.



\section{Fig. 2. ${ }^{99 m}$ Tc-MDP 3-phase bone scan image}

A bone scan image showing a marked increase in the accumulation of radioactivity in the distal phalanx of the left thumb. MDP, methylene diphosphonate.

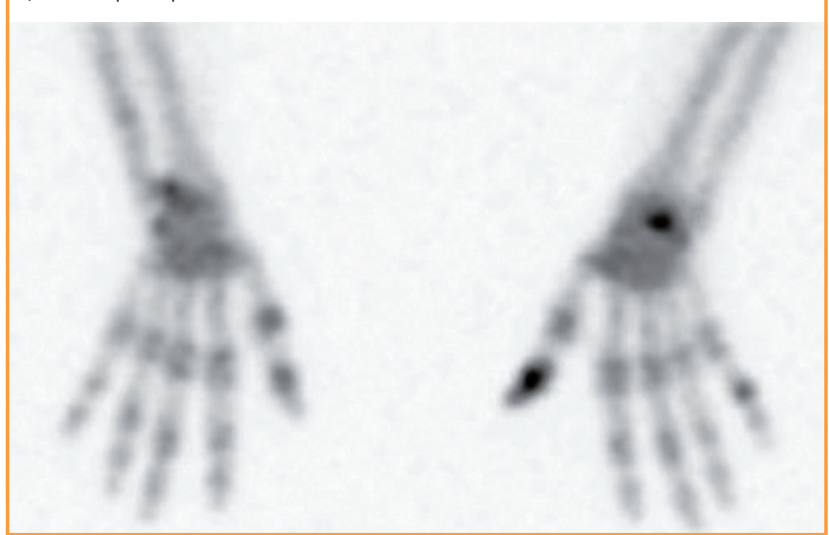

penia at the base of the distal phalangeal bone of her left thumb. With the impression of acute osteomyelitis, a 3-phase bone scan and a magnetic resonance imaging (MRI) examination were performed. The 3-phase bone scan showed a marked accumulation of radioactivity in the distal phalanx of the left thumb (Fig. 2). The MRI exam showed diffuse edema with enhancement of the distal phalanx of the left thumb and the surrounding soft tissue (Fig. 3). The patient, therefore, was diagnosed with acute osteomyelitis in the distal phalangeal bone of the left thumb. She was readmitted and received intravenous antibiotic treatment with moxifloxacin and clindamycin for 5 weeks. She then underwent 7 additional weeks of treatment with the oral forms of the same drugs. At the end of 12 weeks of treatment, a followup 3-phase bone scan was performed, and it showed complete resolution of the osteomyelitis (Fig. 4). At a 2-month follow-up after finishing the course of medication, no complications, such

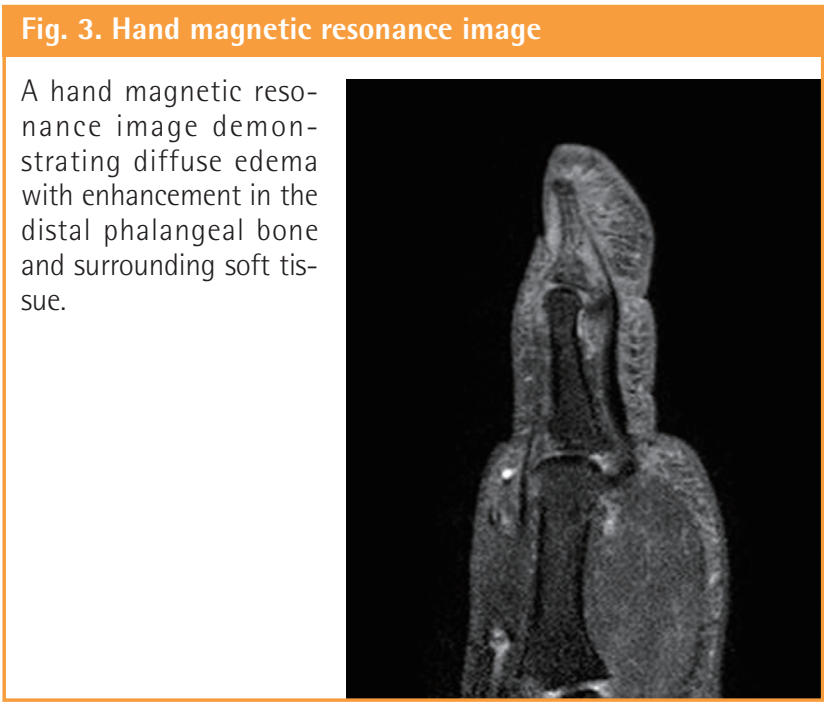

Fig. 4. Follow-up ${ }^{99 m}$ Tc-MDP 3-phase bone scan image

A follow-up bone scan image at 12 weeks after combined intravenous antibiotic treatment showing complete resolution of the accumulation of radioactivity in the left thumb. MDP, methylene diphosphonate.

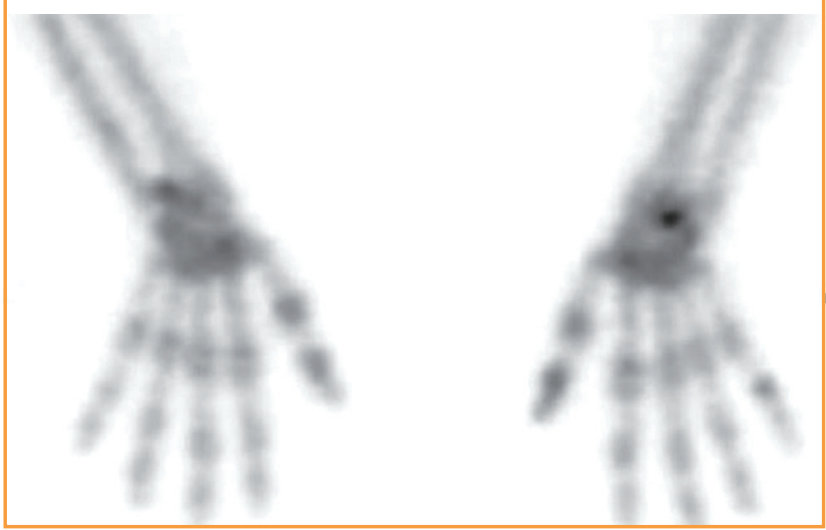


as tenderness, erythema, or swelling, were found.

\section{Case 2}

A 34-year-old male patient visited our emergency center after he was bitten on his right thumb by his pet dog (a Maltese). His distal phalanx was diagnosed with an open fracture, and after open reduction and primary closure of the wound, he was discharged for follow-up. After 3 days, skin necrosis on his right thumb was observed (Fig. 5). He was hospitalized in our department and his wound became aggravated. Ten days after the injury, debridement and open reduction were performed under general anesthesia. Three weeks after injury, a 3-phase bone scan and a MRI examination were performed to evaluate whether osteomyelitis was present. The 3-phase bone scan showed a marked accumulation of radioactivity in the distal phalanx of the right thumb (Fig. 6). The MRI examination showed osteolysis with

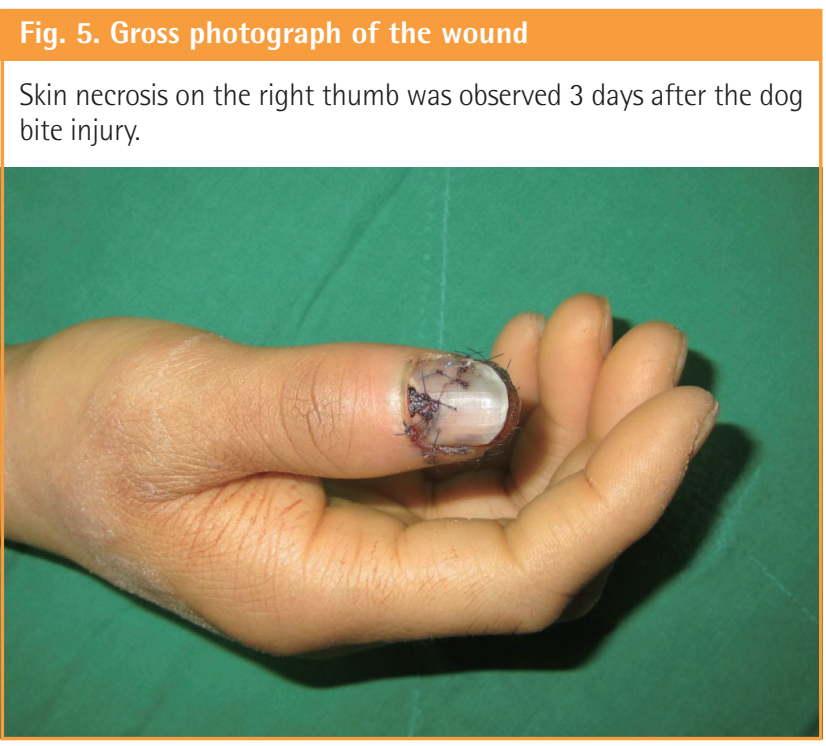

\section{Fig. 6. ${ }^{99 m}$ Tc-MDP 3-phase bone scan image}

A bone scan image showing a marked increase in the accumulation of radioactivity in the distal phalanx of the right thumb. MDP, methylene diphosphonate.

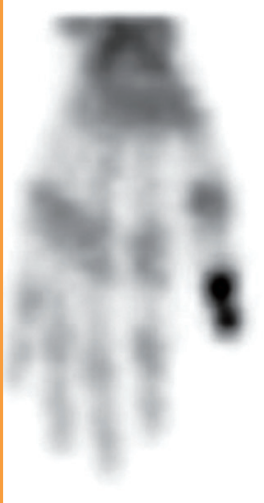

enhancement of the distal phalangeal bone of his right thumb and the surrounding soft tissue (Fig. 7). Intravenous antibiotic treatment with moxifloxacin and clindamycin was administered for 5 weeks, and 7 additional weeks of treatment with the oral forms of the same drugs were prescribed. After 12 months, a follow-up 3-phase bone scan showed complete resolution of the osteomyelitis, and the finger-tip contour was excellent, without joint stiffness or limitation of joint movement (Fig. 8).

\section{Case 3}

A 43-year-old female patient visited our clinic with a laceration wound (approximately $1 \mathrm{~cm}$ ) on her right thenar area due to a bite injury by pet dog (an American Eskimo). The physical examination showed erythema around the wound with mild tenderness and pus-like discharge. She was admitted, and incision and drainage were performed under local anesthesia. A 3-phase

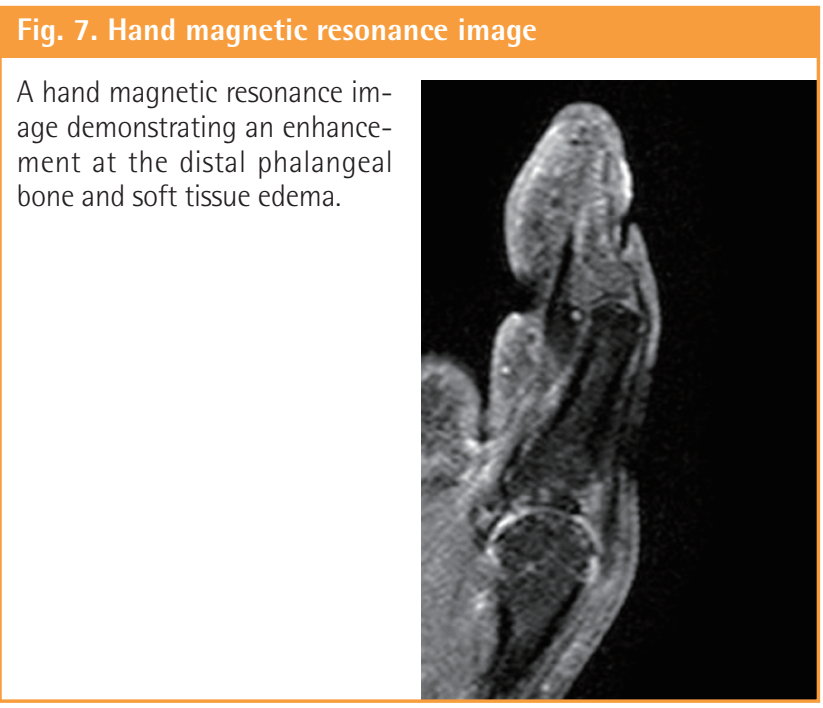

Fig. 8. Follow-up ${ }^{99 m}$ Tc-MDP 3-phase bone scan image

A follow-up ${ }^{99 m}$ Tc-MDP 3-phase bone scan image taken 12 months after treatment, showing complete resolution of the accumulation of radioactivity in the right thumb. MDP, methylene diphosphonate.
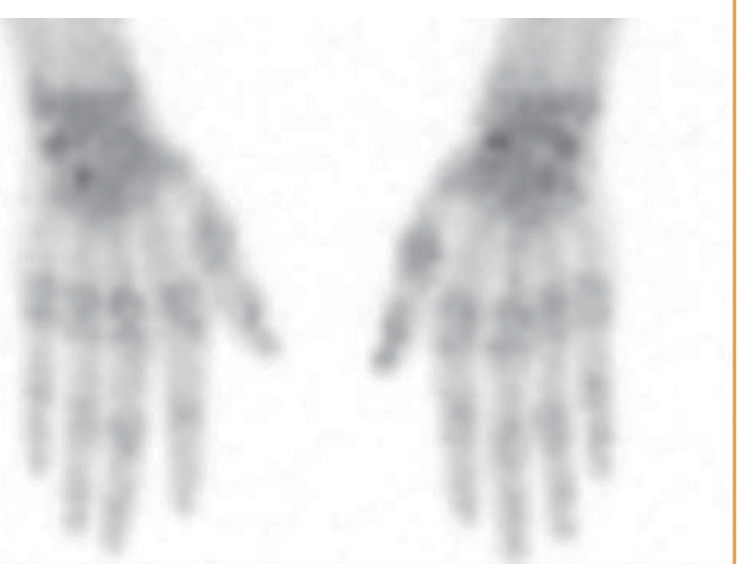
bone scan examination was performed, and the exam revealed a marked accumulation of radioactivity at the shaft of the first metacarpal bone. With the impression of acute osteomyelitis, intravenous antibiotic treatment with moxifloxacin and clindamycin was administered for 5 weeks. The patient also underwent additional 7 weeks of treatment with the oral forms of the same drugs. After the antibiotic treatment was completed, a follow-up 3-phase bone scan was performed, complete resolution of the osteomyelitis was confirmed, and there were no complications such as tenderness or swelling.

\section{DISCUSSION}

Dog bite injuries are common, and most such injuries are simple lacerations or abrasion wounds that may only require primary closure. Some complications may appear after dog bite injuries, and cellulitis is a well-known complication. Nonetheless, osteomyelitis is a rare complication following a dog bite injury [5]. Osteomyelitis has occasionally been reported after cat bite injuries, mainly because cats' teeth tend to be smaller and sharper than dogs' teeth, meaning that they can easily penetrate and injure the periosteum. If osteomyelitis develops secondary to a dog bite injury, it tends to take a more extensive pathologic course and is more difficult to treat than osteomyelitis resulting from cat bite injuries [6]. Moreover, it is difficult to diagnose osteomyelitis, since its latency period is approximately 2 weeks and only half of the Gram-stain wound culture exams reveal pathogenic bacteria [7]. Therefore, a careful examination of the wound is essential during the initial assessment, and even though the initial wound culture may not reveal a common pathogen, such as Pasteurella sp. or Staphylococcus sp., physicians should consider the possibility of infection [8].

When acute osteomyelitis is suspected, follow-up examinations using X-rays, MRI, and 3-phase bone scans should be performed. A simple X-ray examination is the most commonly used modality because it is easy to perform and has a low cost, but X-ray examinations lead to challenges in detecting abnormalities caused by osteomyelitis until about 2 weeks after initial infection, when nearly $50 \%$ of the bone mineral content has been lost, as X-rays have low sensitivity and specificity in the diagnosis of osteomyelitis [9]. Three-phase technetium-99 bone scans have a relatively low cost and are usually positive within a few days of the onset of symptoms, showing a sensitivity of $85 \%$ [9]. However, the specificity is approximately $25 \%$ for the diagnosis of osteomyelitis, and it is hard to pinpoint the infected lesion using this modality [10]. MRI can detect osteomyelitis within 3-5 days of disease onset and has outstanding sensitivity and specificity (as high as 90\%) [9]. Its use can be limited, how- ever, due to its high cost.

We reported 3 cases of acute osteomyelitis that occurred solely due to a dog bite injury. Such cases have rarely been described in the literature, and tend to take a more extensive pathologic course than cases of osteomyelitis due to cat bite injuries $[5,6]$. These cases were treated successfully with proper systemic antibiotics, and in order to confirm the complete recovery from osteomyelitis during the follow-up period, we decided to conduct an objective examination rather than subjective examinations, such as those based on residual symptoms observed by the patient or a physician's observatory opinions. To confirm the complete recovery of a lesion during long-term follow-up, we recommend a 3-phase bone scan examination because its sensitivity is as high as that of MRI, but it has a relatively low cost [9].

Even in minor dog bite injuries, when initial wound cultures and X-ray examinations fail to reveal any abnormalities, the possibility of developing abnormal symptoms exists, and when acute osteomyelitis is suspected, as in our cases, follow-up examinations using X-ray imaging, MRI, and 3-phase bone scans should be performed as soon as possible. In addition, broadspectrum and empiric antibiotic treatment should be administered for approximately $8-12$ weeks before the results of the wound culture are available [11]. Thus, osteomyelitis can be diagnosed and appropriately treated without serious complications.

\section{PATIENT CONSENT}

The patient provided written informed consent for the publication and the use of their images.

\section{REFERENCES}

1. Griego RD, Rosen T, Orengo IF, et al. Dog, cat, and human bites: a review. J Am Acad Dermatol 1995;33:1019-29.

2. Garcia VF. Animal bites and Pasturella infections. Pediatr Rev 1997;18:127-30.

3. Snyder C. Animal bite infections of the hand. Hand Clin 1998;14:691-711.

4. Wiggins ME, Akelman E, Weiss AP. The management of dog bites and dog bite infections to the hand. Orthopedics 1994;17:617-23.

5. Jarvis WR, Banko S, Snyder E, et al. Pasteurella multocida: osteomyelitis following dog bites. Am J Dis Child 1981;135: 625-7.

6. Weber DJ, Wolfson JS, Swartz MN, et al. Pasteurella multocida infections: report of 34 cases and review of the literature. Medicine (Baltimore) 1984;63:133-54. 
7. Chen WL, Chang WN, Chen YS, et al. Acute communityacquired osteoarticular infections in children: high incidence of concomitant bone and joint involvement. J Microbiol Immunol Infect 2010;43:332-8.

8. Presutti RJ. Prevention and treatment of dog bites. Am Fam Physician 2001;63:1567-72.

9. Pineda C, Espinosa R, Pena A. Radiographic imaging in osteomyelitis: the role of plain radiography, computed tomography, ultrasonography, magnetic resonance imaging, and scintigraphy. Semin Plast Surg 2009;23:80-9.

10. Termaat MF, Raijmakers PG, Scholten HJ, et al. The accuracy of diagnostic imaging for the assessment of chronic osteomyelitis: a systematic review and meta-analysis. J Bone Joint Surg Am 2005;87:2464-71.

11. Freshwater A. Why your housecat's trite little bite could cause you quite a fright: a study of domestic felines on the occurrence and antibiotic susceptibility of Pasteurella multocida. Zoonoses Public Health 2008;55:507-13. 